\title{
Bleomycin inhibits proliferation and induces apoptosis in TPC-1 cells through reversing M2-macrophages polarization
}

\author{
HONGWEI LIU ${ }^{1,2}$, HUILEI DONG ${ }^{1,2}$, LI JIANG ${ }^{1,2}$, ZHENDONG LI $^{1,2}$ and XIANDE MA ${ }^{3}$ \\ ${ }^{1}$ Department of Head and Neck Surgery, Cancer Hospital of China Medical University; ${ }^{2}$ Department of Head and \\ Neck Surgery, Liaoning Cancer Hospital and Institute, Shenyang, Liaoning 110042; ${ }^{3}$ College of Integrated \\ Traditional Chinese and Western Medicine, Liaoning University of Traditional \\ Chinese Medicine, Shenyang, Liaoning 110847, P.R. China
}

Received January 3, 2018; Accepted July 3, 2018

DOI: $10.3892 / \mathrm{ol} .2018 .9103$

\begin{abstract}
Papillary thyroid carcinoma (PTC) is one of the most common types of thyroid malignancy. Previous studies have demonstrated that the density of tumor-associated macrophages (TAMs) within the tumor microenvironment affects the progression of PTC due to the imbalance in M1/M2 macrophage subtypes. M2 macrophages induce anti-inflammatory effects and promote tumor progression, whereas M1 macrophages destroy tumor cells. Therefore, reversing TAM polarization to M1 may be a novel strategy for the treatment of cancer. Although bleomycin (BLM) is a commonly used anti-cancer drug, which regulates the secretion of relevant cytokines, high dose and long-term treatment with BLM may lead to pulmonary fibrosis. In the present study, flow cytometry data revealed that low dose treatment with BLM (5 or $10 \mathrm{mU} / \mathrm{ml}$ ) facilitated the expression of the M1 phenotype markers cluster of differentiation (CD)80 and C-C chemokine receptor 7, and concurrently suppressed the M2 marker CD206 on M2-macrophages. Reverse transcription-quantitative polymerase chain reaction data revealed that the expression levels of tumor necrosis factor- $\alpha$ and interleukin-1 $\beta$ markedly increased, whereas the expression of IL-10 decreased in M2 macrophages treated with BLM. A fluorescein isothiocyanate-dextran uptake experiment revealed that BLM increased the phagocytic capacity of M2, however not M1 or M0 macrophages. In addition, to verify the
\end{abstract}

Correspondence to: Dr Zhendong Li, Department of Head and Neck Surgery, Cancer Hospital of China Medical University, 44 Xiaoheyan Road, Dadong, Shenyang, Liaoning 110042, P.R. China

E-mail: 1349946150@qq.com

Dr Xiande Ma, College of Integrated Traditional Chinese and Western Medicine, Liaoning University of Traditional Chinese Medicine, 79 Chongshan Road, Shenyang, Liaoning 110847, P.R. China

E-mail: 15640235233@163.com

Key words: TPC-1, bleomycin, tumor-associated macrophages, polarization effect of BLM-treated M2 macrophages on thyroid carcinoma cells, a co-culture system of macrophages and the human PTC cell line TPC-1, was established. BLM-treated M2 macrophages increased the number of cells in early and late apoptosis and inhibited the migration, proliferation and invasion of TPC-1 cells. These results suggest that a low dose and indirect effect of BLM may induce suppressive effects on PTC by selectively reversing M2 macrophage polarization to $\mathrm{M} 1$, which may provide a novel strategy for cancer treatment.

\section{Introduction}

Papillary thyroid carcinoma (PTC) is one of the most common types of thyroid cancer, accounting for $\sim 80 \%$ of cases (1). At present, surgery remains the primary treatment for PTC; however, for patients with tumors $<1 \mathrm{~cm}$, or multifocal, highly aggressive or metastasizing tumors, the outcome of surgery may be unsatisfactory. Therefore, other therapeutic methods are necessary, particularly immunotherapy (2).

Studies have indicated that the tumor microenvironment, which is necessary for maintaining tumor growth, is comprised of a variety of non-malignant stromal cells. Macrophages within the tumor microenvironment, termed tumor-associated macrophages (TAMs), are some of its most important components (3). The presence of TAMs is associated with the invasion, angiogenesis, hypoxia and early metastasis of tumors, and the suppression of adaptive immunity in various types of tumor, including PTC (4-6). Evidence from clinical and epidemiological studies also indicates a strong association between the increased density of TAMs and a poor prognosis in various kinds of cancer, including breast cancer, ovarian cancer, glioma, lymphoma and PTC (6-8). Commonly, TAMs are subdivided into two types, including the classically activated type 1 macrophages (M1) and the alternatively activated type 2 macrophages (M2), which are distributed throughout PTC tissues $(9,10)$. The M1 phenotype macrophages are activated by interferon (IFN)- $\gamma$, lipopolysaccharides (LPS) and tumor necrosis factor- $\alpha$ (TNF- $\alpha$ ) and are characterized by the production of pro-inflammatory cytokines, including TNF- $\alpha$, interleukin (IL)-1 $\beta$, IL-6, IL-12 and inducible nitric oxide synthase (iNOS). Furthermore, this phenotype is associated with an extended survival time in patients with non-small-cell 
lung cancer $(11,12)$. However, M2 macrophages, described as inhibitors of inflammation, are associated with tumor initiation and progression $(11,12)$. Anti-inflammatory cytokines produced by M2 macrophages, including IL-10, can reduce the expression of iNOS, and inhibit antigen presentation and T cell proliferation $(13,14)$. Furthermore, M2 macrophages are responsible for the growth and survival of various tumor cells $(15,16)$. TAMs within a number of types of malignant cancer predominantly exhibit an M2-like phenotype, which promotes tumor growth and progression by stimulating tumor cell proliferation; therefore, inhibiting the functions of TAMs will inhibit tumorigenesis $(6,17-19)$. Thus, it is hypothesized that the reversal of the polarization of TAMs may be a novel direction for the treatment of inoperable PTCs.

Bleomycin (BLM), a mixture of cytotoxic glycopeptide antibiotics isolated from Streptomyces verticillus, is widely used for anti-tumor treatment, including testicular cancer, malignant lymphoma, head and neck squamous cell carcinoma, cervical cancer and skin cancer (20-23). As BLM does not induce pronounced hepatic, renal or bone marrow toxicity, it is usually used in combination with other anticancer drugs $(24,25)$. However, high dose, long-term treatment with BLM can lead to pulmonary fibrosis $(26,27)$ through the induction of DNA damage $(28,29)$. Previous studies have demonstrated that BLM can stimulate the production of proinflammatory cytokines (IL-18, IL-6 and IL-1 $\beta$ ) and chemokines in THP-1 acute monocytic leukemia cells by activating Toll-like receptor (TLR)2 (30-32), suggesting that BLM may potentially enhance the anti-tumor role of macrophages and regulate their polarization. To verify the effect of low dose BLM treatment on macrophage polarization and the resulting anti-cancer effects, a model of human macrophage polarization was established using THP-1 cells. It was determined that BLM may inhibit the proliferation and invasion of TPC-1 cells, and facilitate apoptosis via promoting the transition of M2 phenotype macrophages to the M1-like phenotype.

\section{Materials and methods}

Reagents. Recombinant human (rh)IL-4, rhIFN- $\gamma$ and rhIL-13 were purchased from R\&D Systems, Inc., (Minneapolis, MN, USA). Phorbol myristate acetate (PMA), BLM and LPS were supplied by Sigma-Aldrich; Merck KGaA (Darmstadt, Germany). BLM solutions were prepared with endotoxin-free saline to a final concentration of $10 \mathrm{U} / \mathrm{ml}$ and stored at $-20^{\circ} \mathrm{C}$. The anti-human-CD14-peridinin chlorophyll protein complex (PerCP; cat. no: 340585), CD68-fluorescein isothiocyanate (FITC; cat. no: 562117), CD80-allophycocyanin (APC; cat. no: 561134), CCR7-phycoerythrin (PE)-cyanine (cy)7(cat. no: 560922), CD206-PE(cat. no: 555954) and atched isotype antibodies were all supplied by BD Biosciences (Franklin Lakes, NJ, USA). FITC-dextran was purchased from Sigma-Aldrich; Merck KGaA.

Cell culture and macrophage polarization. THP-1 human monocyte cells and TPC-1 human thyroid carcinoma cells were cultured in RPMI 1640 medium (Invitrogen; Thermo Fisher Scientific, Inc., Waltham, MA, USA) supplemented with $10 \%$ heat-inactivated fetal bovine serum (Invitrogen; Thermo Fisher Scientific, Inc.) and incubated at $37^{\circ} \mathrm{C}$ in an atmosphere with $5 \% \mathrm{CO}_{2}$. THP-1 cells were induced to differentiate into an attached macrophage-like phenotype by stimulation with $100 \mathrm{nM}$ PMA for $24 \mathrm{~h}$. Subsequently, the cells were cultured in serum-free RPMI-1640 for an additional $24 \mathrm{~h}$ to eliminate the effects of PMA. The attached THP-1 cells, which corresponded to M0 macrophages, were polarized to $\mathrm{M} 1$ by adding $1 \mu \mathrm{g} / \mathrm{ml} \mathrm{LPS}$ and $20 \mathrm{ng} / \mathrm{ml} \mathrm{rhIFN}-\gamma$, or to M2 by adding $20 \mathrm{ng} / \mathrm{ml} \mathrm{rhIL}-4$ and $20 \mathrm{ng} / \mathrm{ml} \mathrm{rhIL}-13$. Serum-free medium was added for a further $24 \mathrm{~h}$ to eliminate the effects of cytokines. The resulting types of macrophages were used in the subsequent experiments.

BLM cytotoxicity assay against MO macrophages. The cytotoxic effect of BLM on M0 macrophages was analyzed by a lactate dehydrogenase (LDH) cytotoxicity assay kit (Beyotime Institute of Biotechnology, Haimen, China) according to the manufacturer's protocol. Briefly, $2 \times 10^{4} \mathrm{M} 0$ macrophages were incubated with various concentrations $(5,10,15,20$ and $25 \mathrm{mU} / \mathrm{ml}$ ) of BLM. Supernatant was collected after $24 \mathrm{~h}$ and $\mathrm{LDH}$ release was quantified. Absorbance was measured at $490 \mathrm{~nm}$ by a microplate reader. All cell culture experiments were performed in duplicate and repeated five times.

Co-culture of TPC-1 cells with THP-1 macrophages. BLM (5 or $10 \mathrm{mU} / \mathrm{ml}$ ) was added to successfully induced M0, M1 or M2 macrophages for 3 days. Subsequently, 5,000 cells TPC-1 cells and macrophages were co-cultured in a Transwell apparatus (pore size, $0.4 \mu \mathrm{m}$; Costar; Corning Incorporated, Corning, NY, USA) for 3 days.

Flow cytometry. The expression of the cell surface markers CD68, CD14, CD206, CD80 and CCR7 was used to determine the macrophage subtypes using a flow cytometer (FACS Canto II; BD Biosciences). For the apoptosis assays, TPC-1 cells were analyzed by flow cytometry using an Annexin V-FITC kit (Beyotime Institute of Biotechnology)-based assay according to the manufacturer's protocol. For the phagocytosis assay, macrophages were incubated with FITC-dextran at $4^{\circ} \mathrm{C}$ and $37^{\circ} \mathrm{C}$, and analyzed by flow cytometry. The phagocytosis rate was determined as follows: Phagocytosis of cells $(\%)=$ Phagocytosis of cells at $37^{\circ} \mathrm{C}(\%)$-Phagocytosis of cells at $4^{\circ} \mathrm{C}(\%)$.

Reverse transcription-quantitative polymerase chain reaction $(R T-q P C R)$. Total RNA was extracted using the RNeasy mini kit with DNase (Qiagen GmbH, Hilden, Germany) according to the manufacturer's protocol. Total RNA $(2 \mu \mathrm{g})$ was reverse-transcribed using a Transcriptor first strand cDNA synthesis kit (Roche Diagnostics, Basel, Switzerland). SYBR Green qPCR was performed using an ABI Prism 7900HT sequence detection system (Applied Biosystems; Thermo Fisher Scientific, Inc.). The PCR primers were 5'-GAGGCT ACGGCGCTGTCA-3' (forward) and 5'-TCCACGGCCTTG CTCTTG-3' (reverse) for IL-10; 5'-CAGTGGCAATGAGGA TGACTTG-3' (forward) and 5'-AGTGGTGGTCGGAGA TTCGT-3' (reverse) for IL-1 $\beta$; 5'-CTTCTGCCTGCTGCA CTTTG-3' (forward) and 5'-GGCCAGAGGGCTGATTAG AGA-3' (reverse) for TNF- $\alpha$; and 5'-GGTGAAGGTCGGTGT GAACG-3' (forward) and 5'-CTCGCTCCTGGAAGATGG TG-3' (reverse) for GADPH. The fold-change for each gene 
was calculated using the $2^{-\Delta \Delta \mathrm{Cq}}$ method (33), and the expression levels were normalized to GADPH.

Wound-healing and proliferation assays. For in vitro wound-healing assays, wounds were made in confluent monolayers of TPC-1 cells using a pipette tip, and images were captured following co-culture with macrophages for $12 \mathrm{~h}$. For cell proliferation assays, TPC-1 cells were co-cultured with macrophages at $37^{\circ} \mathrm{C}$ for 1,3 and 5 days. MTT assays were performed at each time point using an MTT kit (Beyotime Institute of Biotechnology, Shanghai, China) according to the manufacturer's protocol.

Cell invasion assays. Assays were performed in 24-well plates using a Transwell apparatus (pore size, $8 \mu \mathrm{m}$; Costar; Corning Incorporated). A total of 5,000 TPC-1 cells were seeded in serum-free medium in the upper chamber and 50,000 macrophages were seeded in the lower chamber with medium containing $10 \%$ serum. After incubation for $24 \mathrm{~h}$ at $37^{\circ} \mathrm{C}$, cells in the upper chamber were carefully removed with a cotton swab, and lower chamber cells were fixed in methanol and stained with crystal violet. Images were captured under a microscope and cells were counted in 5 randomly selected fields of view.

Statistical analysis. Flow cytometry data analysis was performed using FlowJo software v.7.6 (Tree Star, Inc., Ashland, OR, USA). All data were analyzed by one-way analysis of variance (bonfferoni: compare all pairs of columns) or Mann-Whitney test (non-parametric) using GraphPad Prism v.5.0 software (GraphPad Software, Inc, La Jolla, CA, USA). $\mathrm{P}<0.05$ was considered to indicate a statistically significant difference.

\section{Results}

PMA promotes the differentiation of human THP-1 monocytes into MO phenotype macrophages. Differentiation of THP-1 monocytes has been widely used as an in vitro model of human macrophages. Light microscopy demonstrated that THP-1 cells exhibited a round shape and a non-adherent growth pattern (Fig. 1A), whereas following treatment with PMA for $24 \mathrm{~h}$, cells became adherent with the typical flat, amoeboid-shaped, elongated and branching macrophage morphology (Fig. 1A). The macrophage-like THP-1 cells, hereafter designated as M0 macrophages, exhibited the downregulation of CD14 and the upregulation of CD68 compared with untreated THP-1 cells (Fig. 1B and C); this result is consistent with previous reports $(33,34)$. In addition, the majority of M0 macrophages expressed the CD68 marker, indicating that CD68 may be a suitable marker for M0 macrophages. Thus, THP-1 monocytes were successfully differentiated into M0 macrophages by treatment with PMA.

$B L M$ reverses the phenotype of $M 2$ macrophages to $M 1$. THP-1-dervied M0 macrophages were used to further determine the effects of BLM on macrophage polarization. Firstly, an LDH cytotoxicity assay was performed using M0 macrophages to determine the optimal concentration of BLM. The results demonstrated that BLM at doses lower than $15 \mathrm{mU} / \mathrm{ml}$ induced
A

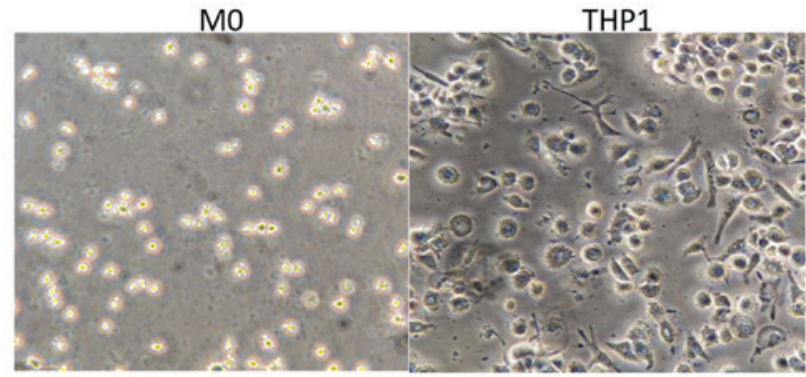

B

Count
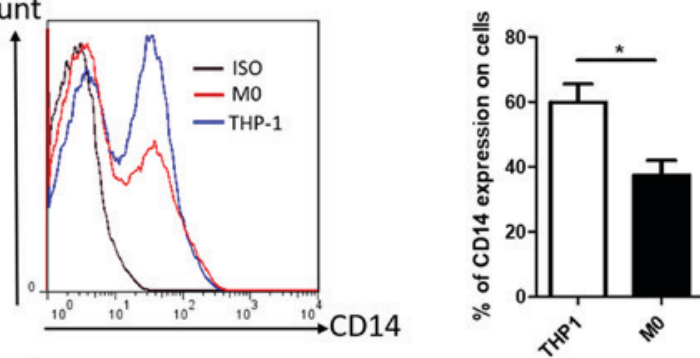

C Count
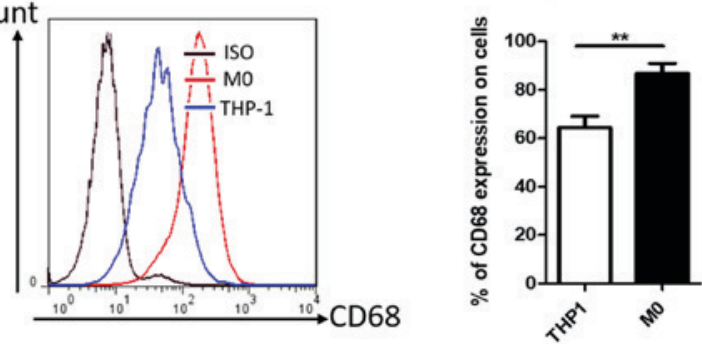

Figure 1. PMA promotes the differentiation of human THP-1 monocytes into macrophages. (A) Representative images of THP-1 cells and PMA-derived M0 macrophages. Magnification, x200. The expression levels of M0 macrophage markers (B) CD14 and (C) CD68 were analyzed by flow cytometry. The left-side panels are representative flow cytometry histograms showing the frequencies of CD14 and CD68 expression on the THP-1 cells and M0 macrophages, respectively. Black lines indicate the isotype controls, red lines represent M0 macrophages and blue lines represent THP-1 cells. The right-side panels in (B) and (C) present the percentage of CD14 and CD68 expression on THP-1 cells and M0 macrophages, respectively. ${ }^{*} \mathrm{P}<0.05$ and ${ }^{* *} \mathrm{P}<0.01$. PMA, phorbol myristate acetate; $\mathrm{CD}$, cluster of differentiation.

no significant cytotoxic effects on M0 macrophages. However, significant cytotoxic effects were observed when BLM was administered at a concentration of 20 or $25 \mathrm{mU} / \mathrm{ml}$ (Fig. 2A). Therefore, low doses of BLM (5 or $10 \mathrm{mU} / \mathrm{ml}$ ) were selected for further experiments. Following a previously described protocol (35,36), M1 and M2 macrophages were successfully induced from M0 macrophages by incubation with LPS and rhIFN- $\gamma$, or rhIL-4 and rhIL-13, for $24 \mathrm{~h}$. The phenotype markers CD80 and CCR7 indicated the successful induction of the M1 phenotype, while the CD206 marker indicated the presence of M2 macrophages (Fig. 2B and C). When 5 or $10 \mathrm{mU} / \mathrm{ml}$ BLM was incubated with M0 or M1 macrophages for a further $24 \mathrm{~h}$, no notable alterations of CD80, CCR7 and CD206 were detected (Fig. 2C). However, when M2 macrophages received the same treatment, the expression levels of CD80 and CCR7 markedly increased, while CD206 was downregulated (Fig. 2B and C), indicating that low dose BLM treatment could reverse M2 macrophages to M1.

$B L M$ reverses the function of $M 2$ macrophages into $M 1$. TAMs regulate immune and inflammatory responses in the tumor microenvironment through the secretion of cytokines (37). M1 
A

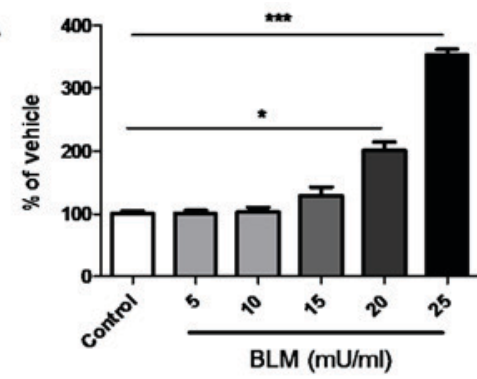

B
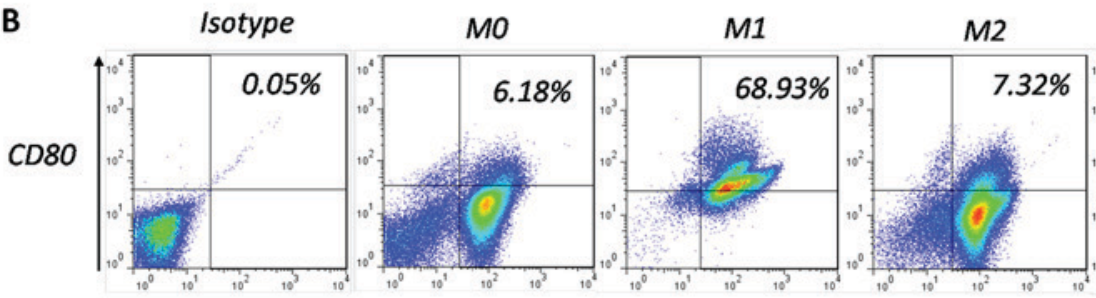

$M 2+B L M-5 \mathrm{mU} / \mathrm{ml} \quad \mathrm{M} 2+B L \mathrm{M}-10 \mathrm{mU} / \mathrm{ml}$
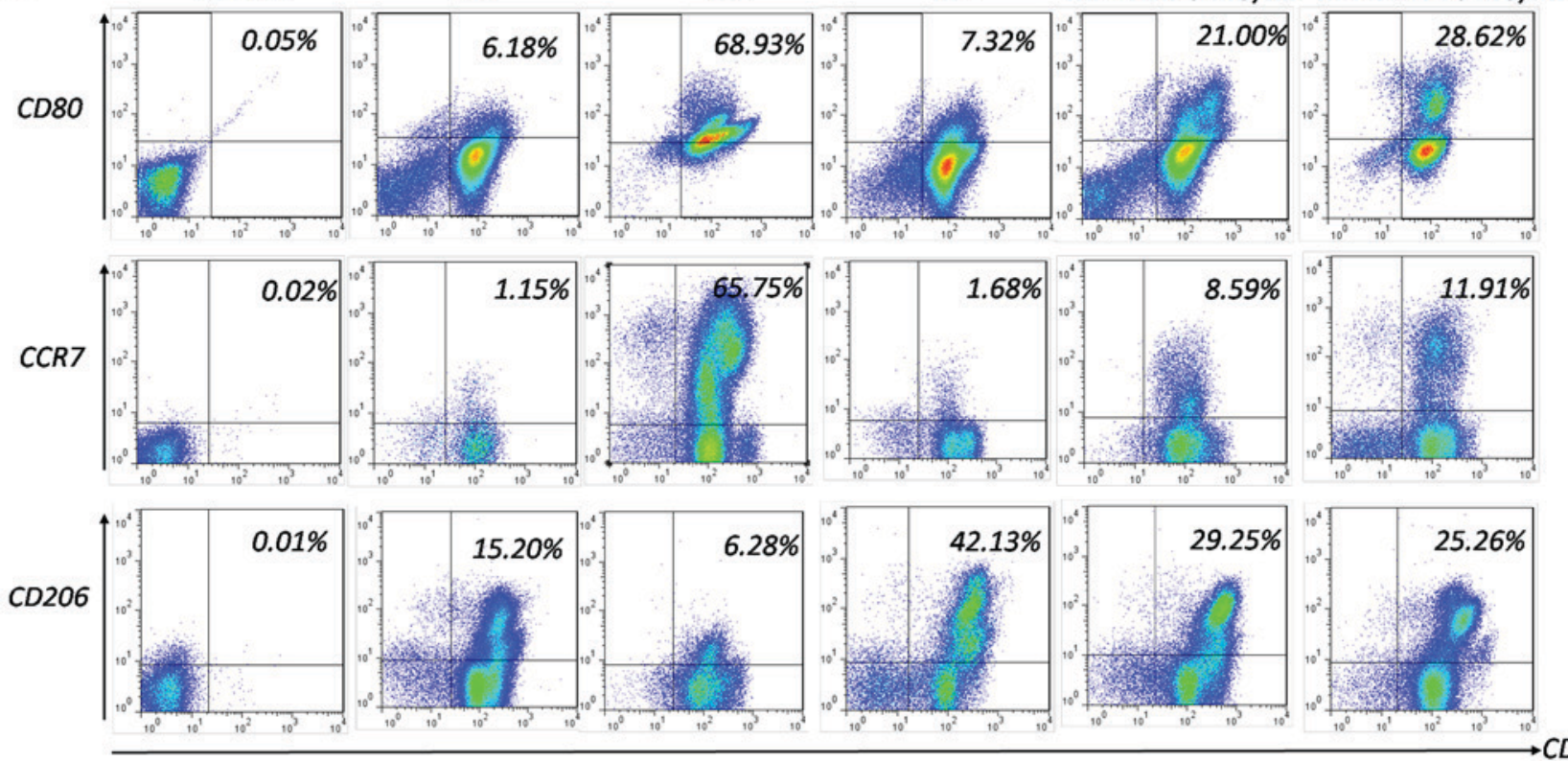

C
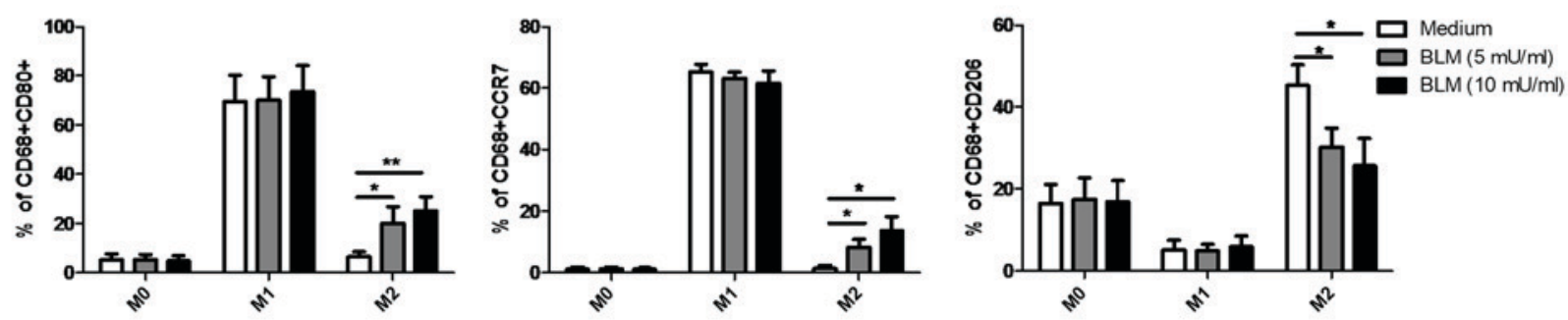

Figure 2. BLM reverses the M2 macrophage phenotype to M1. (A) Lactate dehydrogenase cell cytotoxicity assay of phorbol myristate acetate-derived M0 macrophages cultured with vehicle or BLM treatment $(1,2.5,5,7.5$ and $10 \mathrm{mU} / \mathrm{ml})$. (B) Flow cytometry histogram and (C) bar graph to demonstrate the percentage of CD80, CCR7 and CD206 expression on CD68 macrophages. CD80 and CCR7 are specific surface markers for M1 macrophages, and CD206 is a specific surface marker for $\mathrm{M} 2$ macrophages. ${ }^{*} \mathrm{P}<0.05,{ }^{* *} \mathrm{P}<0.01$ and ${ }^{* * * *} \mathrm{P}<0.001$. BLM, bleomycin; $\mathrm{CD}$, cluster of differentiation; CCR, C-C chemokine receptor.

macrophages primarily secrete pro-inflammatory cytokines (TNF- $\alpha$, IL-1 $\beta$ and IL-6), while M2 macrophages secrete anti-inflammatory cytokines (IL-10 and TGF- $\beta$ ) (37). To further observe the effects of BLM on cytokine secretion in macrophages, the cytokine mRNA levels were measured in all types of macrophages following treatment with 5 or $10 \mathrm{mU} / \mathrm{ml}$ BLM for $24 \mathrm{~h}$. As presented in Fig. 3A-C, the expression of TNF- $\alpha$, IL-1 $\beta$ and IL-10 in M0 and M1 macrophages was not affected by treatment with BLM. However, the expression levels of TNF- $\alpha$ and IL- $1 \beta$ markedly increased and the expression of IL-10 decreased in M2 macrophages in a concentration-dependent manner following treatment with BLM (Fig. 3A-C). In addition, phagocytosis, an important function of macrophages, mediates innate immunity and antigen processing. To further determine the effects of BLM on macrophages, the phagocytic capacity of macrophages was detected by flow cytometry. Consistent with the previous data, the results indicated that M2 macrophages exhibited an elevated FITC-dextran uptake capacity following incubation with BLM (Fig. 3D and E). However, the phagocytic capacity of M1 and M0 macrophages treated with BLM remained unaltered (Fig. 3D).

BLM inhibits the migration and proliferation of TPC-1 cells by reversing $M 2$ macrophage polarization. Since M 2 macrophages were previously reported to increase the growth and survival rate of various types of cancer cell $(15,16)$, a co-culture experiment with TPC-1 cells and M2 macrophages was used to study the effects of BLM on the migration and proliferation of TPC-1 cells via reversing M2 macrophage polarization. 
A

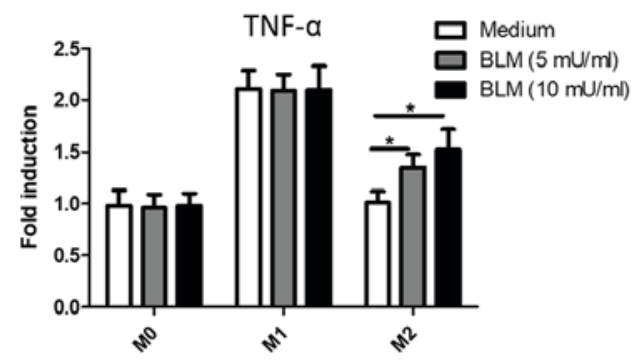

C

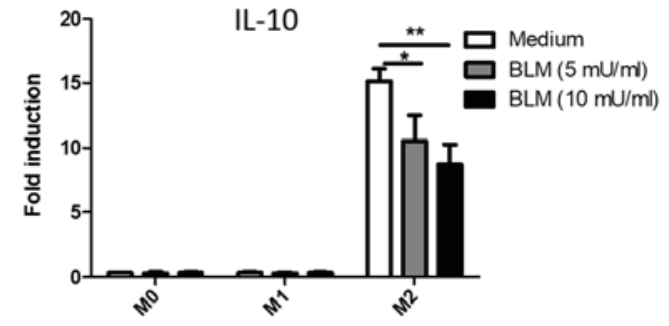

B

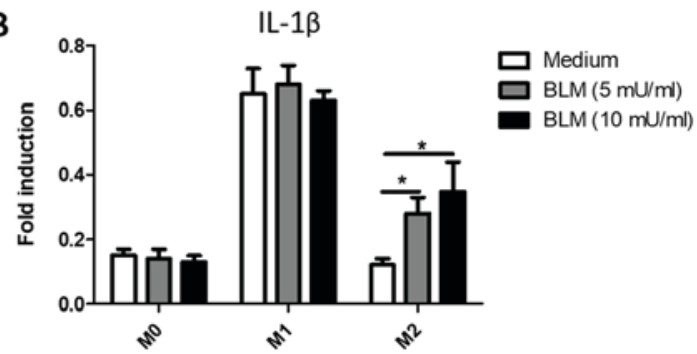

D

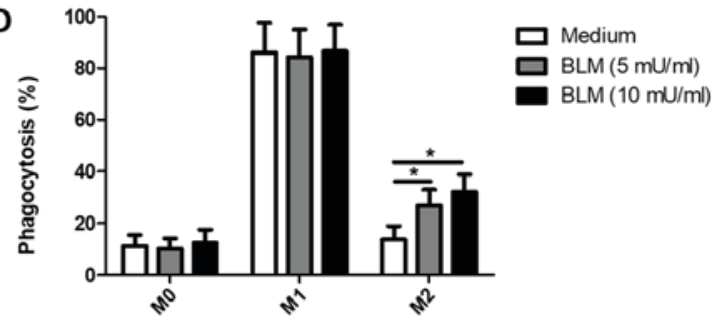

E

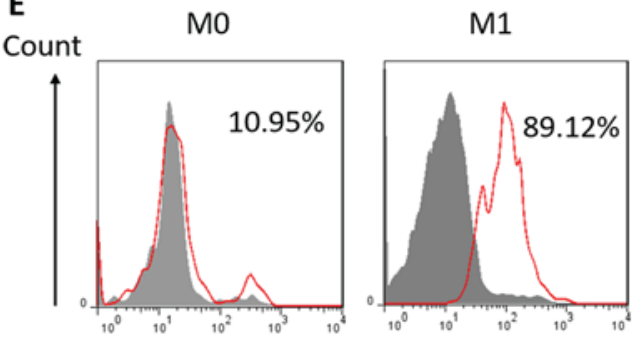

M2

$\mathrm{M} 2+\mathrm{BLM}-5 \mathrm{mU} / \mathrm{ml}$

$\mathrm{M} 2+\mathrm{BLM}-10 \mathrm{mU} / \mathrm{ml}$
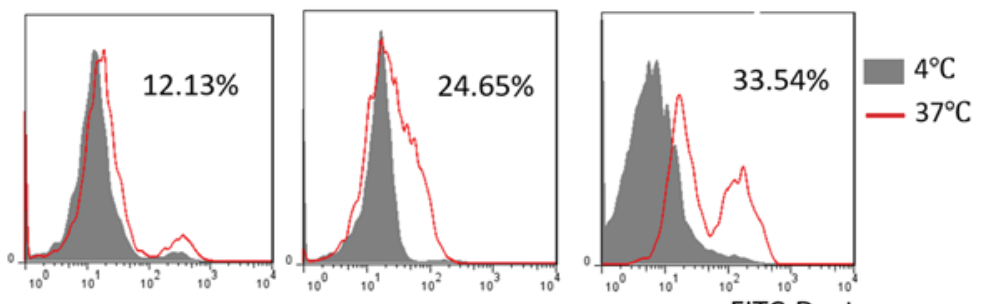

FITC-Dextran

Figure 3. BLM reverses the function of M2 macrophages to M1. Cytokines, including (A) TNF- $\alpha$, (B) IL- $\beta$ and (C) IL-10 were detected by reverse transcription-quantitative polymerase chain reaction. (D) Bar graph and (E) flow cytometry histogram of the rate of fluorescein isothiocyanate-dextran uptake by macrophages. ${ }^{*} \mathrm{P}<0.05$ and ${ }^{* *} \mathrm{P}<0.01$. BLM, bleomycin; TNF- $\alpha$, tumor necrosis factor- $\alpha$; IL, interleukin.

Wound-healing assays indicated that the migration of TPC-1 cells was inhibited following co-culture with BLM-treated M2 macrophages (Fig. 4A). Furthermore, MTT assays demonstrated that co-culture with BLM-treated M2 macrophages inhibited the proliferation of TPC-1 cells compared with M2 macrophages without induction by BLM (Fig. 4B).

$B L M$ inhibits the invasion of TPC-1 cells by reversing $M 2$ macrophage polarization. To determine whether BLM-treated M2 macrophages could regulate the invasion of TPC-1 cells, the number of invading TPC-1 cells was counted following co-culture with BLM-treated M2 macrophages. As presented in Fig. 5, the invasion ability of TPC-1 cells co-cultured with BLM-treated M2 macrophages significantly decreased; however, this effect was not observed following co-culture with BLM-treated M1 and M0 macrophages.

BLM promotes the apoptosis of TPC-1 cells by reversing M2 macrophage polarization. To further verify the anti-tumor effects of BLM-induced M2 macrophage depolarization, the rate of apoptosis induced by co-culture of macrophages with TPC-1 cells was determined. The results indicated that M2 macrophages exhibited a significantly reduced ability to facilitate the early and late apoptosis of TPC- 1 cells $(2.95$ and $1.46 \%$ ) compared with M1 macrophages (10.12 and $13.10 \%$; Fig. 6A). Although the pro-apoptotic ability of M2 cells treated with either 5 or $10 \mathrm{mU} / \mathrm{ml}$ BLM was weaker compared with M1 macrophages, the early apoptosis rates

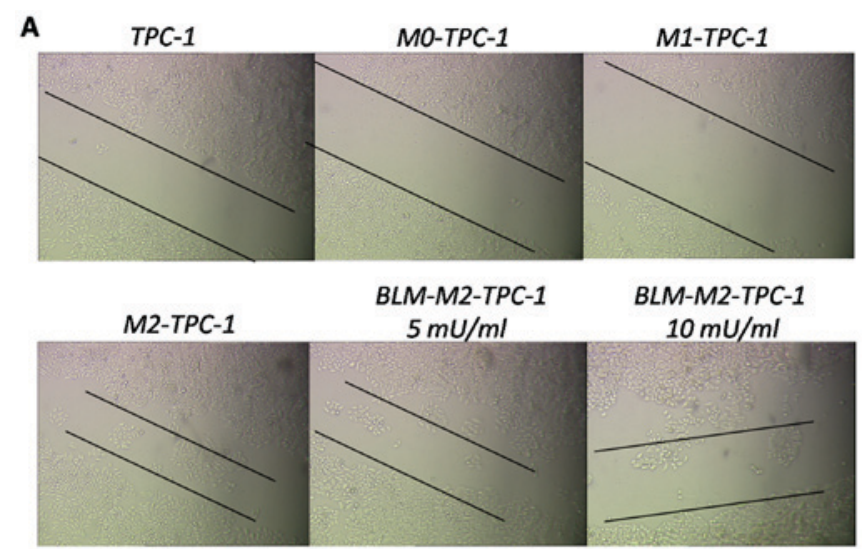

B

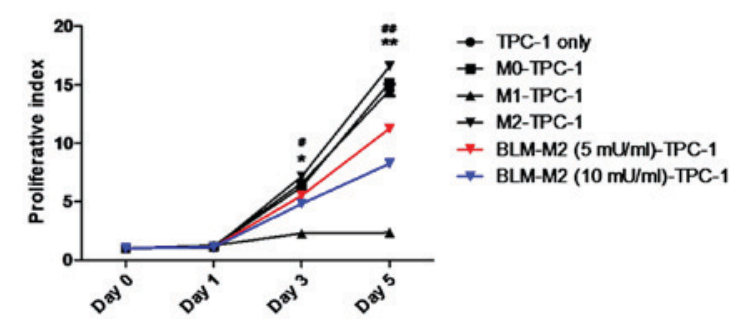

Figure 4. BLM inhibits migration and proliferation of TPC-1 cells by reversing M2 macrophage polarization. (A) Wound-healing assays with TPC-1 cells following co-culture with BLM-treated macrophages for $12 \mathrm{~h}$. Magnification, x40. (B) MTT assays were performed for TPC-1 cells following co-culture with BLM-treated macrophages for $0,1,3$ and 5 days. ${ }^{*} \mathrm{P}<0.05$ and ${ }^{* *} \mathrm{P}<0.01$ vs. $5 \mathrm{mU} / \mathrm{ml} \mathrm{BLM}$ treatment; ${ }^{\#} \mathrm{P}<0.05$ and ${ }^{\# \#} \mathrm{P}<0.01$ vs. $10 \mathrm{mU} / \mathrm{ml}$ BLM treatment. BLM, bleomycin. 
A
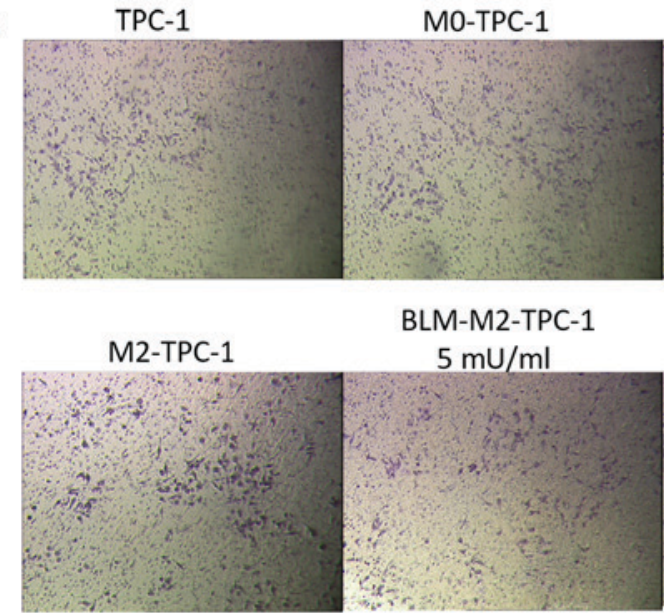

BLM-M2-TPC-1 $5 \mathrm{mU} / \mathrm{ml}$
M1-TPC-1

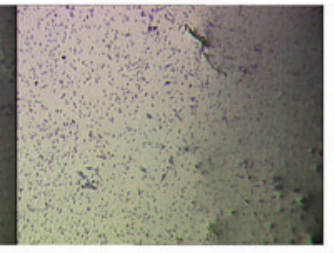

BLM-M2-TPC-1 $10 \mathrm{mU} / \mathrm{ml}$

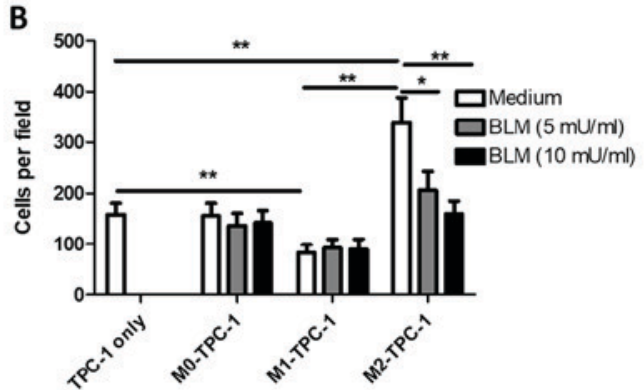

Figure 5. BLM inhibits the invasion of TPC-1 cells by reversing M2-macrophage polarization. (A) The invasion ability of TPC-1 cells was detected following co-culture with macrophages treated with 5 or $10 \mathrm{mU} / \mathrm{ml}$ BLM. Images were captured under a microscope (magnification, x100). (B) Number of cells per field of view. ${ }^{*} \mathrm{P}<0.05$ and ${ }^{* *} \mathrm{P}<0.01$. BLM, bleomycin.

A

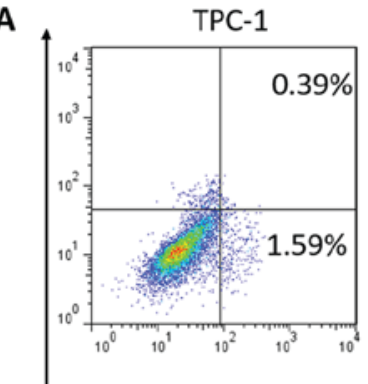

$\mathrm{Pl}$

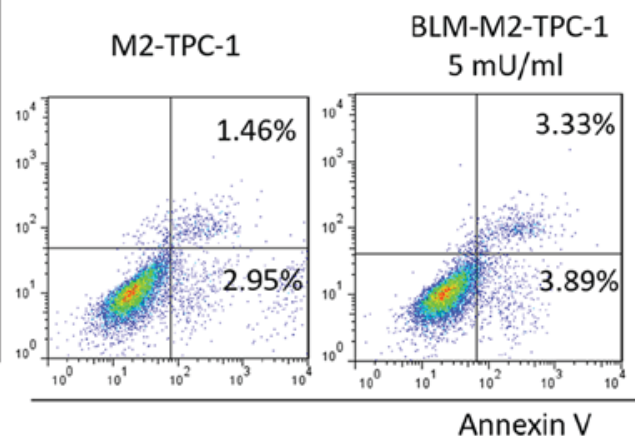

MO-TPC-1
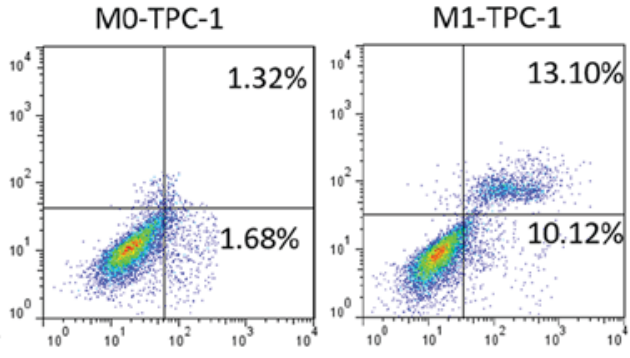

BLM-M2-TPC-1 $10 \mathrm{mU} / \mathrm{ml}$

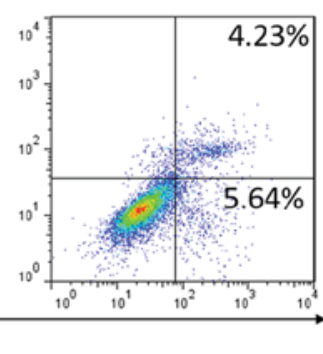

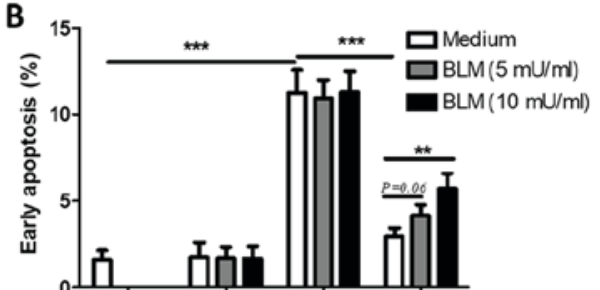
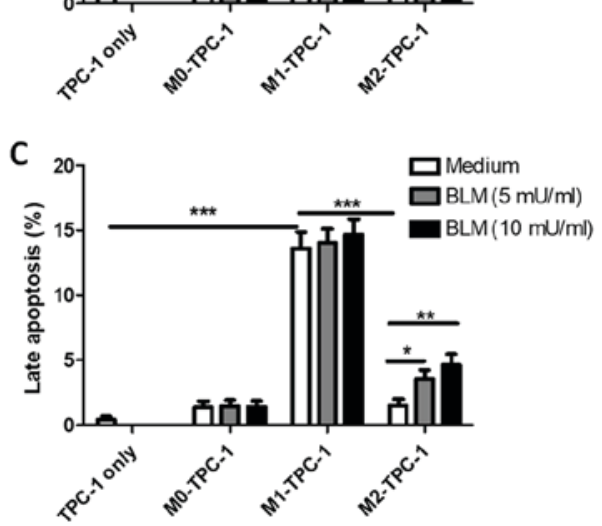

Figure 6. BLM promotes the apoptosis of TPC-1 cells by reversing M2-macrophage polarization. (A) Subsequent to the co-culture of TPC-1 cells and macrophages for $48 \mathrm{~h}$ in a Transwell apparatus (pore size, $0.4 \mu \mathrm{m}$ ), an AnnexinV-FITC apoptosis detection kit was used for apoptosis detection. AnnexinV ${ }^{+} \mathrm{PI}$ indicated early apoptosis and $\mathrm{AnnexinV}^{+} \mathrm{PI}^{+}$indicated late apoptosis. ( $\mathrm{B}$ and $\mathrm{C}$ ) Bar charts of the quantified rate of apoptosis. ${ }^{*} \mathrm{P}<0.05$, ${ }^{* *} \mathrm{P}<0.01$ and ${ }^{* * * *} \mathrm{P}<0.001$. BLM, bleomycin.

were significantly upregulated compared with untreated M2 macrophages (Fig. 6A-C). Furthermore, BLM-treated M1 or M0 macrophages did not affect the early or late apoptosis of TPC-1 cells (Fig. 6B and C).

\section{Discussion}

TAMs have been proposed to promote tumor progression by facilitating angiogenesis, lymphogenesis, stroma remodeling and immune suppression in various types of cancer (36,38-40). TAMs also serve roles in tumor invasion and metastasis by secreting enzymes, including plasmin, urokinase-type plasminogen activator, matrix metalloproteinases and cathepsin B (41-43). An increased density of TAMs in tumor tissues is associated with a poor prognosis (44). TAMs are commonly divided into M1 and M2 subtypes, which exhibit anti-cancer effects, and potentiate tumorigenesis and tumor progression, respectively. Previous studies have indicated that TAMs in numerous types of malignant cancer predominantly exhibit an M2-like phenotype. Since M2 macrophages in tumor tissue contribute to an immunosuppressive microenvironment and fuel tumor progression, reversing M2-TAM polarization could be a novel therapeutic strategy against cancer (45). In the present study, low doses of BLM could reverse M2 macrophages to an M1-like phenotype, as demonstrated by the upregulation of the M1 surface markers CD80 and CCR7 and the increased expression of predominantly M1-secreted cytokines (TNF- $\alpha$ 
and IL-1 $\beta$ ). Furthermore, BLM could also enhance the phagocytic function of M2 macrophages in a dose-dependent manner. However, the phagocytic capacity was not significantly altered in M1 and M0 macrophages treated with BLM, suggesting that BLM could re-polarize M2 macrophage phenotype to M1 phenotype, regulate the balance of M1/M2 TAMs in the tumor microenvironment and activate the Th1 cell-based anti-cancer immune response $(46,47)$. BLM may induces the tumor suppression ability via selectively reversing M2 macrophages to the tumoricidal M1 phenotype without affecting M1 or M0 macrophages.

The presence of TAMs in tumor cores has been found to be associated with progressive PTC features and TAM-conditioned medium-enhanced PTC cell invasion (9). Furthermore, Kim et al (48) identified that primary PTC tumors with lymph node metastasis and higher TAM density were associated with larger tumor sizes, suggesting a tumorigenic role for TAMs in human PTC (4). Although a number of studies have demonstrated that M2-TAMs induce tumor proliferation, migration and invasion in hepatocellular carcinoma, human basal carcinoma, pancreatic cancer and prostate cancer $(49,50)$, to the best of our knowledge, the reversed polarization of TAMs in the context of PTC was not previously reported. Since BLM induced TAM depolarization, a co-culture system of TPC-1 cells and BLM-treated macrophages was developed in the present study. As expected, the proliferation, migration and invasion of TPC-1 cells were inhibited following co-culture with BLM-treated M2 macrophages, indicating that BLM could inhibit PTC progression by regulating TAM polarization.

It was previously demonstrated that the tumor suppression capacity of M1 macrophages is associated with the activation of the NF- $\mathrm{kB}$ signaling pathway (51), which serves a key role in modulating the apoptosis of tumor cells. The present study also indicated that BLM-induced M1-like macrophages facilitated both early and late apoptosis of TPC-1 cells. However, it was previously reported that BLM can directly induce cancer cell apoptosis and inhibit proliferation (51-54), and the proposed mechanism was through affecting the $\mathrm{G}_{2}$ and $\mathrm{M}$ phase cell cycle progression of cancer cells $(55,56)$. Furthermore, an increased expression of p21, independent of $\mathrm{p} 53$, was identified in fibrotic lungs following direct induction with BLM (57). Recent data also revealed the crucial roles of $\mathrm{NF}-\kappa \mathrm{B}$ and $\mathrm{p} 21$ in the $\mathrm{p} 53$-independent apoptosis of cancer cells, in response to DNA-damaging drugs $(51,52)$. The interaction between the functions of p21 and mediators involved in macrophage differentiation, including NF- $\mathrm{kB}$, could be an important consideration in attempt to elucidate the apoptotic cell response to BLM. Nevertheless, the results of the present study indicated that BLM could indirectly inhibit TPC-1 cell proliferation and induce apoptosis by depolarizing M2 macrophages to M1 macrophages. This result is supported by previous reports that $\mathrm{M} 1$ macrophages inhibit cell proliferation and induce apoptosis (58-60). BLM is widely used for tumor treatment, but high dose and long-term exposure to BLM may lead to pulmonary fibrosis due to DNA damage $(27,28)$. In the present study, tumor suppression was indirectly induced by a low dose of BLM though the effect on macrophages.

In conclusion, the results indicate that low dose, indirect treatment with BLM can suppress PTC by selectively reversing
M2 macrophage polarization to M1. However, further studies are necessary to identify the signaling pathways induced by BLM in TAM polarization, which may contribute to improved treatment methods for a variety of cancer types.

\section{Acknowledgements}

Not applicable.

\section{Funding}

The present study was finically supported by the Beijing CSCO Clinical Cancer Research Foundation (grant no. 201510784).

\section{Availability of data and materials}

The analyzed data sets generated during the study are available from the corresponding author, on reasonable request.

\section{Authors' contributions}

LH, LZ and MX designed the study. LH, DH and MX performed the experiments. LH, JL and LZ analyzed the data.

\section{Ethics approval and consent to participate}

Not applicable.

\section{Patient consent for publication}

Not applicable.

\section{Competing interests}

The authors declare that they have no competing interests.

\section{References}

1. Cancer Genome Atlas Network: Integrated genomic characterization of papillary thyroid carcinoma. Cell 159: 676-690, 2014.

2. Walker S: Immunotherapy and the treatment of non-small cell lung cancer. J Adv Pract Oncol 7: 304-306, 2016.

3. Pusztaszeri MP, Faquin WC and Sadow PM: Tumor-associated inflammatory cells in thyroid carcinomas. Surg Pathol Clin 7: 501-514, 2014.

4. Qing W, Fang WY, Ye L, Shen LY, Zhang XF, Fei XC, Chen X, Wang WQ, Li XY, Xiao JC and Ning G: Density of tumor-associated macrophages correlate with lymph node metastasis in papillary thyroid carcinoma. Thyroid 22, 905-910, 2012.

5. Quatromoni JG and Eruslanov E: Tumor-associated macrophages: Function, phenotype and link to prognosis in human Lung Cancer. Am J Transl Res 4: 376-389, 2012.

6. Mantovani A, Sozzani S, Locati M, Allavena P and Sica A: Macrophage polarization: Tumor-associated macrophages as a paradigm for polarized M2 mononuclear phagocytes. Trends Immunol 23: 549-555, 2002.

7. Allavena P, Sica A, Solinas G, Porta C and Mantovani A: The inflammatory micro-environment in tumor progression: The role of tumor-associated macrophages. Crit Rev Oncol Hematol 66: $1-9,2008$.

8. Can NY, Ayturk S, Celik M, Sezer YA, Ozyilmaz F, Tastekin E, Sut N, Ustun F, Bulbul BY, Puyan FO and Guldiken S: Histological perspective on the effects of tumor-associated macrophages in the tumor microenvironment surrounding papillary thyroid carcinoma. Pol J Pathol 67: 332-344, 2016. 
9. Fang W, Ye L, Shen L, Cai J, Huang F, Wei Q, Fei X, Chen X, Guan H, Wang W, et al: Tumor-associated macrophages promote the metastatic potential of thyroid papillary cancer by releasing CXCL8. Carcinogenesis 35: 1780-1787, 2014.

10. Chang WC, Chen JY, Lee CH and Yang AH: Expression of decoy receptor 3 in diffuse sclerosing variant of papillary thyroid carcinoma: Correlation with M2 macrophage differentiation and lymphatic invasion. Thyroid 23: 720-726, 2013

11. Lopez-Gonzalez JS, Avila-Moreno F, Prado-Garcia H, Aguilar-Cazares D, Mandoki JJ and Meneses-Flores M: Lung carcinomas decrease the number of monocytes/macrophages (CD14+ cells) that produce TNF-alpha. Clin Immunol 122: 323-329, 2007.

12. Ohri CM, Shikotra A, Green RH, Waller DA and Bradding P: Macrophages within NSCLC tumour islets are predominantly of a cytotoxic M1 phenotype associated with extended survival. Eur Respir J 33: 118-126, 2009.

13. Redente EF, Dwyer-Nield LD, Merrick DT, Raina K, Agarwal R, Pao W, Rice PL, Shroyer KR and Malkinson AM: Tumor progression stage and anatomical site regulate tumor-associated macrophage and bone marrow-derived monocyte polarization. Am J Pathol 176: 2972-2985, 2010

14. Zea AH, Rodriguez PC, Atkins MB, Hernandez C, Signoretti S, Zabaleta J, McDermott D, Quiceno D, Youmans A, $\mathrm{O}$ 'Neill A, et al: Arginase-producing myeloid suppressor cells in renal cell carcinoma patients: A mechanism of tumor evasion. Cancer Res 65: 3044-3048, 2005.

15. Chang CI, Liao JC and Kuo L: Macrophage arginase promotes tumor cell growth and suppresses nitric oxide-mediated tumor cytotoxicity. Cancer Res 61: 1100-1106, 2001

16. Biswas SK, Sica A and Lewis CE: Plasticity of macrophage function during tumor progression: Regulation by distinct molecular mechanisms. J Immunol 180: 2011-2057, 2008.

17. Qian BZ and Pollard JW: Macrophage diversity enhances tumor progression and metastasis. Cell 141: 39-51, 2010.

18. Mehrpour M, Esclatine A, Beau I and Codogno P: Overview of macroautophagy regulation in mammalian cells. Cell Res 20 748-762, 2010

19. Ruffell B, Affara NI and Coussens LM: Differential macrophage programming in the tumor microenvironment. Trends Immunol 33: 119-126, 2012.

20. Batschinski K, Dervisis N, Kitchell B, Newman R and Erfourth T: Combination of bleomycin and cytosine arabinoside chemotherapy for relapsed canine lymphoma. J Am Anim Hosp Assoc 54: 150-155, 2018.

21. Ng K, Duncan S, Shamash J and Alifrangis C: Dose intense chemotherapy in the management of poor prognosis and relapsed testicular cancer: Experiences and controversies. Expert Rev Anticancer Ther 18: 431-436, 2018.

22. Porwal PK, Dubey KP, Morey A, Singh H, Pooja S and Bose A: Bleomycin sclerotherapy in lymphangiomas of head and neck: prospective study of 8 cases. Indian J Otolaryngol Head Neck Surg 70: 145-148, 2018

23. Racnik J, Svara T,Zadravec M, Gombac M, Cemazar M, Sersa G and Tozon N: Electrochemotherapy with bleomycin of different types of cutaneous tumours in a ferret (Mustela Putorius Furo). Radiol Oncol 52: 98-104, 2017

24. Cai Y, Sun R, Wang R, Ren JG, Zhang W, Zhao YF and Zhao JH: The activation of Akt/mTOR pathway by bleomycin in Epithelial-to-mesenchymal transition of human submandibular gland cells: A treatment mechanism of bleomycin for mucoceles of the salivary glands. Biomed Pharmacother 90: 109-115, 2017.

25. Mouratis MA and Aidinis V: Modeling pulmonary fibrosis with bleomycin. Curr Opin Pulm Med 17: 355-361, 2011.

26. Weng D, Chen JX, Li HH, Liu F, Zhou LD, Liu HP, Zheng RJ, Jiang Y, Liu ZH and Ge B: 2-aminopurine suppresses the TGF- $\beta 1$-induced epithelial-mesenchymal transition and attenuates bleomycin-induced pulmonary fibrosis. Cell Death Discov 4 17, 2018.

27. Jung WJ, Lee SY, Choi SI, Kim BK, Lee EJ, In KH and Lee MG: Toll-like receptor expression in pulmonary sensory neurons in the bleomycin-induced fibrosis model. PLoS One 13: e0193117, 2018.

28. Bolzan AD and Bianchi MS: DNA and chromosome damage induced by bleomycin in mammalian cells: An update. Mutat Res 775: 51-62, 2018

29. Massonneau J, Ouellet C, Lucien F, Dubois CM, Tyler J and Boissonneault G: Suboptimal extracellular $\mathrm{pH}$ values alter DNA damage response to induced double-strand breaks. FEBS Open Bio 8: 416-425, 2018.
30. Huang W, Wang G, Phelps DS, Al-Mondhiry $\mathrm{H}$ and Floros J: Combined SP-A-bleomycin effect on cytokines by THP-1 cells: Impact of surfactant lipids on this effect. Am J Physiol Lung Cell Mol Physiol 283: L94-L102, 2002

31. Razonable RR, Henault M and Paya CV: Stimulation of toll-like receptor 2 with bleomycin results in cellular activation and secretion of pro-inflammatory cytokines and chemokines. Toxicol Appl Pharmacol 210: 181-189, 2006.

32. Hoshino T, Okamoto M, Sakazaki Y, Kato S, Young HA and Aizawa H: Role of proinflammatory cytokines IL-18 and IL-1beta in bleomycin-induced lung injury in humans and mice. Am J Respir Cell Mol Biol 41: 661-670, 2009

33. Genin M, Clement F, Fattaccioli A, Raes M and Michiels C: M1 and M2 macrophages derived from THP-1 cells differentially modulate the response of cancer cells to etoposide. BMC Cancer 15: 577, 2015.

34. Steinbach F and Thiele B: Phenotypic investigation of mononuclear phagocytes by flow cytometry. J Immunol Methods 174: 109-122, 1994

35. Murdoch C, Muthana M, Coffelt SB and Lewis CE: The role of myeloid cells in the promotion of tumour angiogenesis. Nat Rev Cancer 8: 618-631, 2008.

36. Yuan A, Hsiao YJ, Chen HY, Chen HW, Ho CC, Chen YY, Liu YC, Hong TH, Yu SL, Chen JJ and Yang PC: Opposite effects of M1 and M2 macrophage subtypes on lung cancer progression. Sci Rep 5: 14273, 2015.

37. Reeves AR, Spiller KL, Freytes DO, Vunjak-Novakovic G and Kaplan DL: Controlled release of cytokines using silk-biomaterials for macrophage polarization. Biomaterials 73 272-283, 2015.

38. Pang L, Han S, Jiao Y, Jiang S, He X and Li P: Bu Fei Decoction attenuates the tumor associated macrophage stimulated proliferation, migration, invasion and immunosuppression of non-small cell Lung Cancer, partially via IL-10 and PD-L1 regulation. Int J Oncol 51: 25-38, 2017

39. Kang H, Zhang J, Wang B, Liu M, Zhao J, Yang M and Li Y: Puerarin inhibits M2 polarization and metastasis of tumor-associated macrophages from NSCLC xenograft model via inactivating MEK/ERK 1/2 pathway. Int J Oncol 50: 545-554, 2017.

40. Cho SW, Kim YA, Sun HJ, Kim YA, Oh BC, Yi KH, Park DJ and Park YJ: CXCL16 signaling mediated macrophage effects on tumor invasion of papillary thyroid carcinoma. Endocr Relat Cancer 23: 113-124, 2016.

41. Komohara Y, Fujiwara Y, Ohnishi K and Takeya M: Tumor-associated macrophages: Potential therapeutic targets for anti-cancer therapy. Adv Drug Deliv Rev 99: 180-185, 2016.

42. Gocheva V, Wang HW, Gadea BB, Shree T, Hunter KE, Garfall AL, Berman T and Joyce JA: IL-4 induces cathepsin protease activity in tumor-associated macrophages to promote cancer growth and invasion. Genes Dev 24: 241-255, 2010.

43. Wang R, Zhang J, Chen S, Lu M, Luo X, Yao S, Liu S, Qin Y and Chen H: Tumor-associated macrophages provide a suitable microenvironment for non-small Lung Cancer invasion and progression. Lung Cancer 74: 188-196, 2011.

44. Komohara Y, Jinushi M and Takeya M: Clinical significance of macrophage heterogeneity in human malignant tumors. Cancer Sci 105: 1-8, 2014

45. Rolny C, Mazzone M, Tugues S, Laoui D, Johansson I, Coulon C, Squadrito ML, Segura I, Li X, Knevels E, et al: HRG inhibits tumor growth and metastasis by inducing macrophage polarization and vessel normalization through downregulation of PIGF. Cancer Cell 19: 31-44, 2011.

46. Almatroodi SA, McDonald CF, Darby IA and Pouniotis DS Characterization of M1/M2 tumour-associated macrophages (TAMs) and Th1/Th2 cytokine profiles in patients with NSCLC. Cancer Microenviron 9: 1-11, 2016.

47. Muraille E, Leo O and Moser M: TH1/TH2 paradigm extended: Macrophage polarization as an unappreciated pathogen-driven escape mechanism. Front Immunol 5: 603, 2014.

48. Kim S, Cho SW, Min HS, Kim KM, Yeom GJ, Kim EY, Lee KE, Yun YG,Park DJ and Park YJ: The expression of tumor-associated macrophages in papillary thyroid carcinoma. Endocrinol Metab (Seoul) 28: 192-198, 2013

49. Mellor AL and Munn DH: Creating immune privilege: Active local suppression that benefits friends, but protects foes. Nat Rev Immunol 8: 74-80, 2008.

50. Sica A, Schioppa T, Mantovani A and Allavena P: Tumour-associated macrophages are a distinct M2 polarised population promoting tumour progression: Potential targets of anti-cancer therapy. Eur J Cancer 42: 717-727, 2006. 
51. Liu CP, Zhang X, Tan QL, Xu WX, Zhou CY, Luo M, Li X, Huang RY and Zeng X: NF- $\mathrm{B}$ pathways are involved in M1 polarization of RAW 264.7 macrophage by polyporus polysaccharide in the tumor microenvironment. PLoS One 12: e0188317, 2017.

52. Gong H, Cao Y, Han G, Zhang Y, You Q, Wang Y and Pan Y: p53/microRNA-374b/AKT1 regulates colorectal cancer cell apoptosis in response to DNA damage. Int J Oncol 50: 1785-1791, 2017.

53. Yang L, Zhao W, Wei P, Zuo W and Zhu S: Tumor suppressor $\mathrm{p} 53$ induces miR-15a processing to inhibit neuronal apoptosis inhibitory protein (NAIP) in the apoptotic response DNA damage in breast cancer cell. Am J Transl Res 9: 683-691, 2017.

54. Liu JF, Nie XC, Shao YC, Su WH and Ma HY: Bleomycin suppresses the proliferation and the mobility of human gastric cancer cells through the smad signaling pathway. Cell Physiol Biochem 40: 1401-1409, 2016.

55. Gao N, Shang B, Zhang X, Shen C, Xu R, Chen R and He Q: Potent antitumor actions of the new antibiotic boningmycin through induction of apoptosis and cellular senescence. Anticancer Drugs 22: 166-175, 2011.

56. Uchida R, Yokota S and Tomoda H: Structure elucidation of meroterpenoid habiterpenol, a novel abrogator of bleomycin-induced G2 arrest in Jurkat cells, produced by Phytohabitans suffuscus 3787_5. J Antibiot (Tokyo) 67: 783-786, 2014.
57. Wang Q, Cui K, Espin-Garcia O, Cheng D, Qiu X, Chen Z, Moore M, Bristow RG, Xu W, Der S and Liu G: Resistance to bleomycin in cancer cell lines is characterized by prolonged doubling time, reduced DNA damage and evasion of G2/M arrest and apoptosis. PLoS One 8: e82363, 2013.

58. Lofdahl A, Rydell-Tormanen K, Larsson-Callerfelt AK, AUID- and Oho Wenglen C: Pulmonary fibrosis in vivo displays increased p21 expression reduced by 5-HT2B receptor antagonists in vitro-a potential pathway affecting proliferation. Sci Rep 8: 1927, 2018.

59. Park HR, Lee EJ, Moon SC, Chung TW and Kim KJ: Inhibition of lung cancer growth by HangAmDan-B is mediated by macrophage activation to M1 subtype. Oncol Lett 13: 2330-2336, 2017.

60. Li Y, Poppoe F, Chen J, Yu L and Deng F: Macrophages polarized by expression of toxoGR A15II inhibit growth of hepatic carcinoma. Front Immunol 8: 137, 2017.

This work is licensed under a Creative Commons Attribution-NonCommercial-NoDerivatives 4.0 International (CC BY-NC-ND 4.0) License. 